\title{
Integrating Data and Image Domain Deep Learning for Limited Angle Tomography using Consensus Equilibrium
}

\author{
Muhammad Usman Ghani, W. Clem Karl \\ Boston University \\ \{mughani, wckarl\}abu.edu
}

\begin{abstract}
Computed Tomography $(C T)$ is a non-invasive imaging modality with applications ranging from healthcare to security. It reconstructs cross-sectional images of an object using a collection of projection data collected at different angles. Conventional methods, such as FBP, require that the projection data be uniformly acquired over the complete angular range. In some applications, it is not possible to acquire such data. Security is one such domain where non-rotational scanning configurations are being developed which violate the complete data assumption. Conventional methods produce images from such data that are filled with artifacts. The recent success of deep learning (DL) methods has inspired researchers to post-process these artifact laden images using deep neural networks (DNNs). This approach has seen limited success on real CT problems. Another approach has been to pre-process the incomplete data using DNNs aiming to avoid the creation of artifacts altogether. Due to imperfections in the learning process, this approach can still leave perceptible residual artifacts. In this work, we aim to combine the power of deep learning in both the data and image domains through a two-step process based on the consensus equilibrium (CE) framework. Specifically, we use conditional generative adversarial networks (cGANs) in both the data and the image domain for enhanced performance and efficient computation and combine them through a consensus process. We demonstrate the effectiveness of our approach on a real security CT dataset for a challenging $90^{\circ}$ limited-angle problem. The same framework can be applied to other limited data problems arising in applications such as electron microscopy, nondestructive evaluation, and medical imaging.
\end{abstract}

\section{Introduction}

$X$-ray computed tomography (CT) is a fundamental imaging tool for many applications in medical healthcare $[1,2]$, materials science [3, 4], industrial testing [5], and
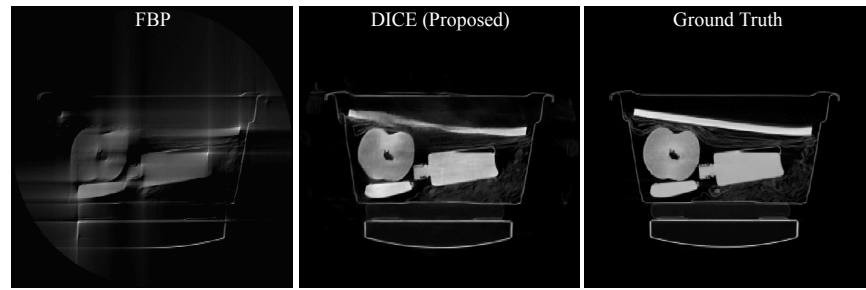

Figure 1. FBP and proposed DICE method reconstructions using $90^{\circ}$ limited angle data are presented along with a reference fullview MBIR reconstruction.

security [6, 7]. It reconstructs cross-sectional images of objects by collecting a series of projection data at all angles and processing the acquired projection data using an image reconstruction algorithm. In a typical 2-D parallelbeam data acquisition setup, data is acquired at all angles $\theta \in\left[0^{0}, 180^{\circ}\right]$. The filtered backprojection algorithm (FBP) is the most widely used algorithm for $\mathrm{CT}$ image reconstruction due to its simple and efficient implementation. An alternative approach is model-based image reconstruction (MBIR) [8], which allows incorporation of both a physical model and prior information about the objects being imaged. MBIR can reconstruct higher quality images as compared to FBP, but its iterative nature makes it computationally very expensive, which has limited its adoption. In certain situations, it becomes impractical to acquire data with full angular coverage, which creates a limited angle CT problem. Conventional, computationally efficient methods such as FBP produce artifact-filled images from such limited angle CT data (see Figure 1), with the severity of the artifacts generally becoming more prominent the more limited the data becomes.

In this paper, we propose to use the consensus equilibrium framework (CE) [9] to integrate prior information from both the data and the image domains along with knowledge of the $X$-ray physics for efficient and improved limited angle CT reconstructions. We encapsulate the prior information from the data and the image domains using deep neural networks (DNNs). In contrast to existing image post-processing-only approaches, our data domain deep 
learning (DL) component learns to complete the projection data and therefore aims to avoid the creation of image domain artifacts. Our image domain DNN learns to improve image quality by learning patch-based priors. Unlike typical image priors such as Total-Variation (TV) [10] or Markov Random Field (MRF) [11], our image domain DNN efficiently learns the salient image features from a large dataset. Moreover, it has been shown in $[12,13]$ that image domain DNN priors can lead to faster convergence. Both of our DNNs are trained using a conditional generative adversarial networks (cGAN) strategy [14]. Overall our proposed method aim to combine the quality benefits of MBIR methods with the flexibility and efficiency of DL methods, combining the data and image domain DL via consensus equilibrium (CE) framework.

\subsection{Motivation and Challenges}

Limited angle CT scans can arise due to the physical limitations of the data acquisition or to achieve other desirable imaging capabilities. For example, acquiring data for a limited number of views could significantly reduce the scan time. This would allow CT imaging of highly dynamic organs in the body such as the heart [15] without introduction of blurring. This would also enable CT based study of physical processes in dynamic objects for materials science applications $[4,16]$. It could also be useful for tomographic imaging of specific regions of interest such as for dental [17] or breast [18] scan. Our own motivation has been checkpoint security where new, non-rotating gantry configurations use a limited number of static $X$-ray sources resulting in a limited angle $\mathrm{CT}$ image formation problem [3].

Deep learning has had great impact in image enhancement in the computer vision community, where the presence of powerful and flexible network models coupled with large datasets [19] and efficient and inexpensive GPU-based computational resources for training have resulted in impressive processing results [14, 20-25]. Motivated by such successes researchers in the tomographic community have rushed to apply these tools to restore the artifact filled images produced by conventional $\mathrm{CT}$ reconstruction methods such as FBP applied to reduced quality CT data. While these DL methods have greatly enhanced $\mathrm{CT}$ reconstruction, they fail to completely solve the problem, especially in severely limited data cases. Relative to medical problem, the problem is even more challenging in the security application, where the underlying scene can have a much larger range of shapes, objects, and materials.

\subsection{Contributions}

The main contributions of this work are summarized below.

- Presentation of a framework to combine data domain and image domain DL in CT image reconstruction.

- Demonstration of the potential of combined data and image DL for limited angle CT arising in security problem that outperforms existing post-processing methods.

- Example images and performance metrics on a real security dataset [26].

\section{Related Work}

Data-driven models have become increasingly popular in image reconstruction research in recent years, including for limited angle CT applications. A recent survey paper by Ravishankar et al. [27] compactly summarizes these advances. Image post-processing using data-derived learned models has been the most popular theme, where a DNN is trained on images directly [28] or in the wavelet domain [29] with the aim of enhancing low-quality FBP-derived images. While these methods can effectively enhance FBP images, they can still fail to recover image features completely; a task which becomes even more challenging in security settings. An alternative approach is to treat the problem in the data domain using a DNN which learns to complete the projection data [30]. However, incorporating projection data consistency conditions is very difficult in DL frameworks [31]. Anirudh et al. proposed an end-to-end DL framework that learns to reconstruct images directly from limited angle data [31]. This approach fails to incorporate the $X$-ray physical model in the learning framework and uses a fully connected layer with a huge number of parameters in the generator network. Wurfl et al. [32] developed a DNN architecture inspired from FBP that learns to adjust projection domain weights to enhance images. This method however has limited flexibility and therefore results in only minor improvements.

A related problem is sparse-view CT which has been widely studied. Fewer projection data are acquired with uniform spacing over complete angular range. Similar approaches have been explored for this sparse-view CT: image post-processing [33-36], projection data completion [3739], combination of projection data completion and image post-processing [40], and end-to-end learning [41, 42].

Another popular theme is regularized inversion with implicitly or explicitly defined priors. The Plug-and-Play priors approach $[43,44]$ does not require priors to be explicitly defined, and therefore allows easy integration of image domain DNNs in a regularized inversion framework [12]. Similar ideas have been explored using other formulations where variable splitting and replacement of proximal operators by learned alternatives is performed [45-48]. The RED method [49] is a similar approach except that it explicitly defines the prior term. 


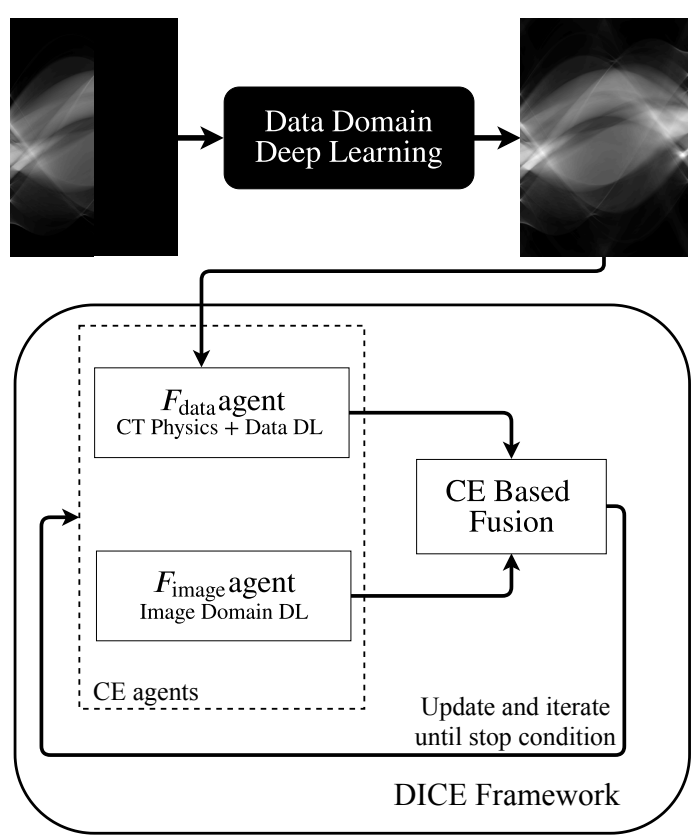

Figure 2. Overview of our DICE framework is presented. Data and image domain DL is integrated into $\mathrm{CT}$ image reconstruction using consensus equilibrium framework.

\section{Integrated Data and Image Domain Deep Learning}

Consensus equilibrium framework [9, 50] was developed as a generalization of the ADMM-derived plug-and-play approach $[43,44]$ as a principled means to integrate multiple heterogenous models or "agents" to yield a single coherent reconstruction. CE starts with a collection of models or agents and derives a consensus solution to the collection. In our case there will be two such agents and the CE equations would be:

$$
\begin{aligned}
F_{\text {data }}\left(v_{1}^{*}=x^{*}+u_{1}^{*}\right) & =x^{*} \\
F_{\text {image }}\left(v_{2}^{*}=x^{*}+u_{2}^{*}\right) & =x^{*} \\
\mu_{1} u_{1}^{*}+\mu_{2} u_{2}^{*} & =0
\end{aligned}
$$

where $x^{*}=\mu_{1} v_{1}^{*}+\mu_{2} v_{2}^{*}$ is the CE estimate of $x$, where $\mu_{i}$ defines the individual contribution of each agent, and $\mathbf{u}$ can be interpreted as noise vector. Intuitively, the functions $F$ are chosen to map initial values of $x$ to improved values and the solution to the CE equations determines a set of inputs $x_{k}$ that balance the forces from the competing agents. See $[9,50]$ for a detailed discussion.

In this work, we combine data and image domain DL for CT image reconstruction using CE and call it DICE framework. An overview of our DICE framework is presented in Figure 2 with major components of our approach. We have two CE agents build around two DL models. The first block combines data domain DL model with CT physics.
The model attempts to complete the limited projection data which is embedded into CT physical model. The second block uses image domain DL and is focused on enhancing tomographic images. The impact of both blocks is combined through the consensus equilibrium (CE) framework, which provides a rational way of combining information from multiple sources. In particular, we construct a $\mathrm{CE}$ formulation consisting of two terms. $F_{\text {data }}$ is CT-physicsderived data consistency constraint and $F_{\text {image }}$ is the DL image domain model. The data consistency term is based on completed data from the data domain DL model. In this way both data domain and image domain DL models are combined for an improved overall outcome. In the following sections we describe each of these pieces.

\subsection{Data Domain Deep Learning}

In this section we describe the data domain DL block in Figure 2. This block is inspired from data domain DL work by Ghani and Karl [38]. The data domain DL block performs the following steps in sequence:

$$
\begin{aligned}
\hat{y}_{\text {complete }} & =\phi_{\text {data }}\left(y_{\text {limited }}\right) \\
\hat{x} & =\operatorname{FBP}\left(\hat{y}_{\text {complete }}\right) \\
\hat{y}_{\text {consistent }} & =\mathbf{A} \hat{x}
\end{aligned}
$$

where $y_{\text {limited }} \in \mathbb{R}^{M / 2}$ is the observed limited angle data, $\phi_{\text {data }}$ is the data domain DNN trained to complete the limited data, $\hat{y}_{\text {complete }} \in \mathbb{R}^{M}$ is the corresponding estimated complete projection data, $\mathbf{A} \in \mathbb{R}^{M \times N}$ is the tomographic forward projection operator, and $\hat{y}_{\text {consistent }} \in \mathbb{R}^{M}$ is the estimated consistent and completed projection data.

Valid tomographic projection data must satisfy a set of consistency conditions [51]. In general, $\hat{y}_{\text {complete, }}$ obtained as the output of our trained DNN, will not satisfy these consistency conditions. While we could simply use this inconsistent data estimate in the following processing, we instead choose to run this projection estimate through an inversion and re-projection cycle to obtain the consistent projection data in $\hat{y}_{\text {consistent }}$. Experience has shown this imposition of data consistency results in improved overall results. Direct mapping of $\hat{y}_{\text {complete }}$ onto the space of consistent projection data is the focus of future work.

The key operation in (4) is the DNN mapping represented by $\phi_{\text {data. }}$. We use a cGAN for our data-domain DNN, which is composed of a generator network which performs data completion coupled with a discriminator network. Isola et al. [14] reported that using such a combination results in better network performance. The generator and discriminator networks in our cGAN is jointly trained by optimizing a mini-max cost function [14, 52]. The overall architecture of our data completion cGAN is presented in Figure 3. Our generator network $\left(\phi_{\text {data }}\right)$ and discriminator $\left(D_{\text {data }}\right)$ both are inspired from $[14,53]$. We 


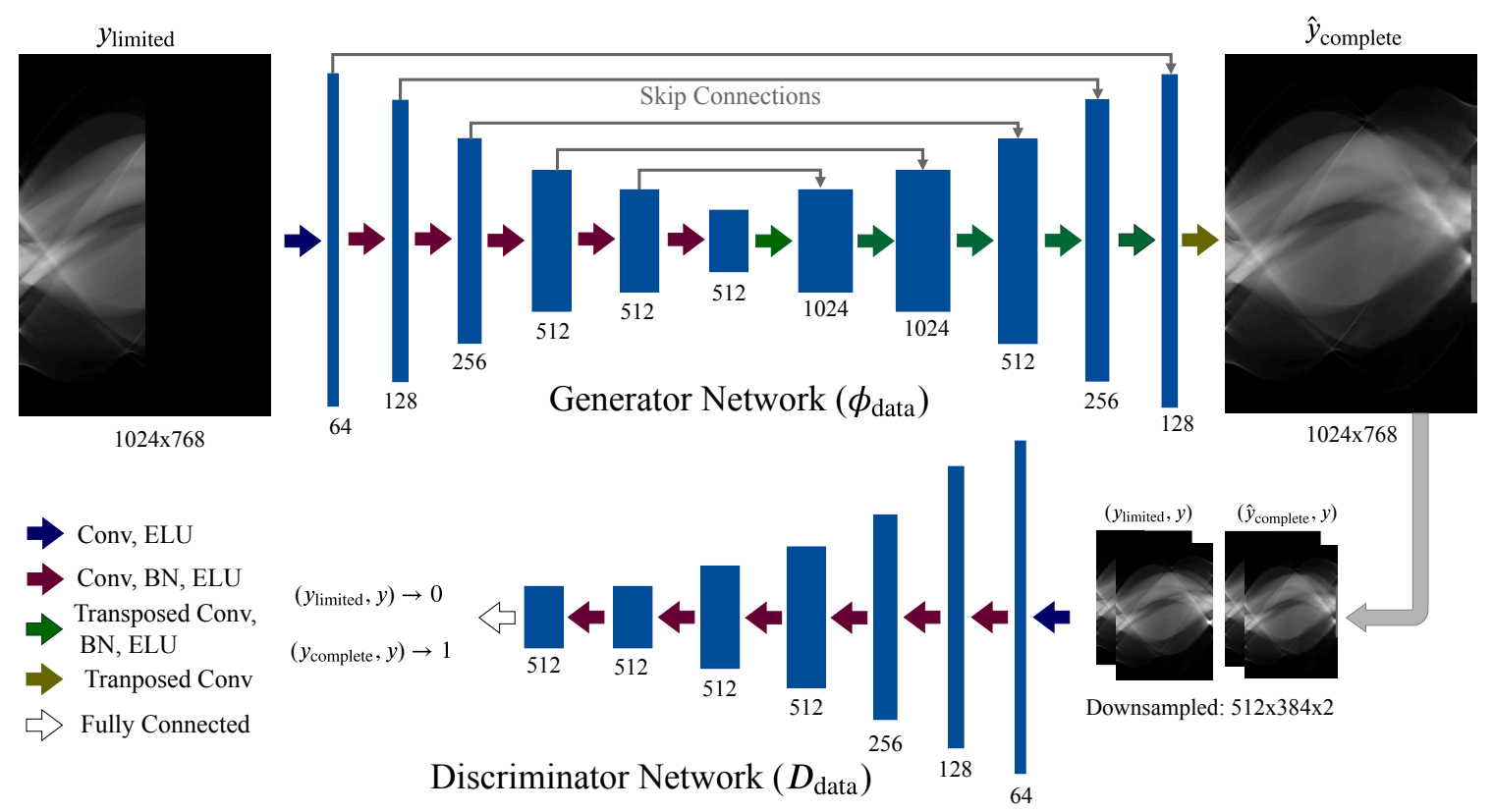

Figure 3. Overall architecture of our data completion cGAN is presented. The abbreviated legends in the Figure are defined here; Conv: 2D convolution, ELU: exponential linear unit, BN: batch-normalization, and 2D Transposed Conv: transposed convolution.

use $7 \times 7$ kernels in the convolutional and transposed convolutional layers in both networks. The convolution and transponsed convolution operations are performed with a 2-pixel stride in both networks. The number of channels used at each layer are given at the bottom of each layer output. The generator network $\phi_{\text {data }}$ has a fully convolutional architecture. It has 6 down-sampling and 6 up-sampling layers. The down-sampling layers use 2-pixel strided convolutions, and the up-sampling layers use 2-pixel strided transponsed convolutions. In addition, the skip connections are used to transport and concatenate high-resolution information from the down-sampling layers to the up-sampling layers. The generator $\phi_{\text {data }}$ has a theoretical effective receptive field $(\mathrm{ERF})$ of $1135 \times 1135$. It is trained to perform blind projection data completion for efficient training, i.e., $\phi_{\text {data }}\left(y_{\text {limited }}\right)=y_{12}$, where $y_{12}$ is output of last layer in $\phi_{\text {data }}$. At test time, we perform mask-specific data completion, i.e., $\phi_{\text {data }}\left(y_{\text {limited }}\right)=y_{\text {limited }}+M \odot y_{12}$, where $M$ is the mask representing missing views and $\odot$ is an elementwise multiplication operation. The discriminator network, $D_{\text {data }}$, has 7 convolutional and a fully connected layer. For efficient training, we use down-sampled projection data as input to $D_{\text {data }}$.

\subsection{Image Domain Deep Learning}

In this section we describe the image domain DL block in Figure 2. Similar to our data-domain DNN, we use a cGAN for $\phi_{\text {image }}$, which is composed of a generator network which performs image enhancement coupled with a discriminator network. The generator and discriminator networks in our cGAN is jointly trained as described earlier. The overall structure of our image domain cGAN $\phi_{\text {image }}$ in presented in Figure 4. The number of channels used at each layer are given at the bottom of each layer output. The inputs of the generator network $\hat{x}$ are patches extracted from lowerquality reconstructions and the ground truth for training $x$ are corresponding patches extracted from full-view MBIR reconstructions [8]. The goal of $\phi_{\text {image }}$ is to encapsulate patch-based image priors by learning low-to-high quality image mappings. The architecture of our image domain generator network $\phi_{\text {image }}$ is inspired from [22]. It is a fully convolutional architecture and follows a residual learning strategy. It has 16 convolutional layers with $5 \times 5$ kernels each performing 1-pixel strided convolutions, resulting in a theoretical ERF of $65 \times 65$. The image discriminator network $D_{\text {image }}$ has 5 convolutional and 2 fully connected layers. The convolutional layers in $D_{\text {image }}$ use $3 \times 3$ kernels and perform 2-pixel strided convolutions.

\subsection{Consensus Equilibrium Framework for Infor- mation Fusion}

We choose the first CE agent to correspond to a standard data fidelity term arising in MAP estimation problems:

$$
F_{\text {data }}\left(v_{1}\right)=\underset{x \geq 0}{\arg \min } \frac{1}{2}\left\|\hat{y}_{\text {consistent }}-\mathbf{A} x\right\|_{W}^{2}+\frac{1}{2 \sigma^{2}}\left\|v_{1}-x\right\|_{2}^{2}
$$

where $W \in \mathbb{R}^{M \times M}$ is a diagonal data weighting matrix with $w_{i} \approx 1 / \operatorname{var}\left(\left[\hat{y}_{\text {consistent }}\right]_{i}\right)$, and $\sigma$ is the trade-off parameter between the two terms. This agent serves to incorporate 


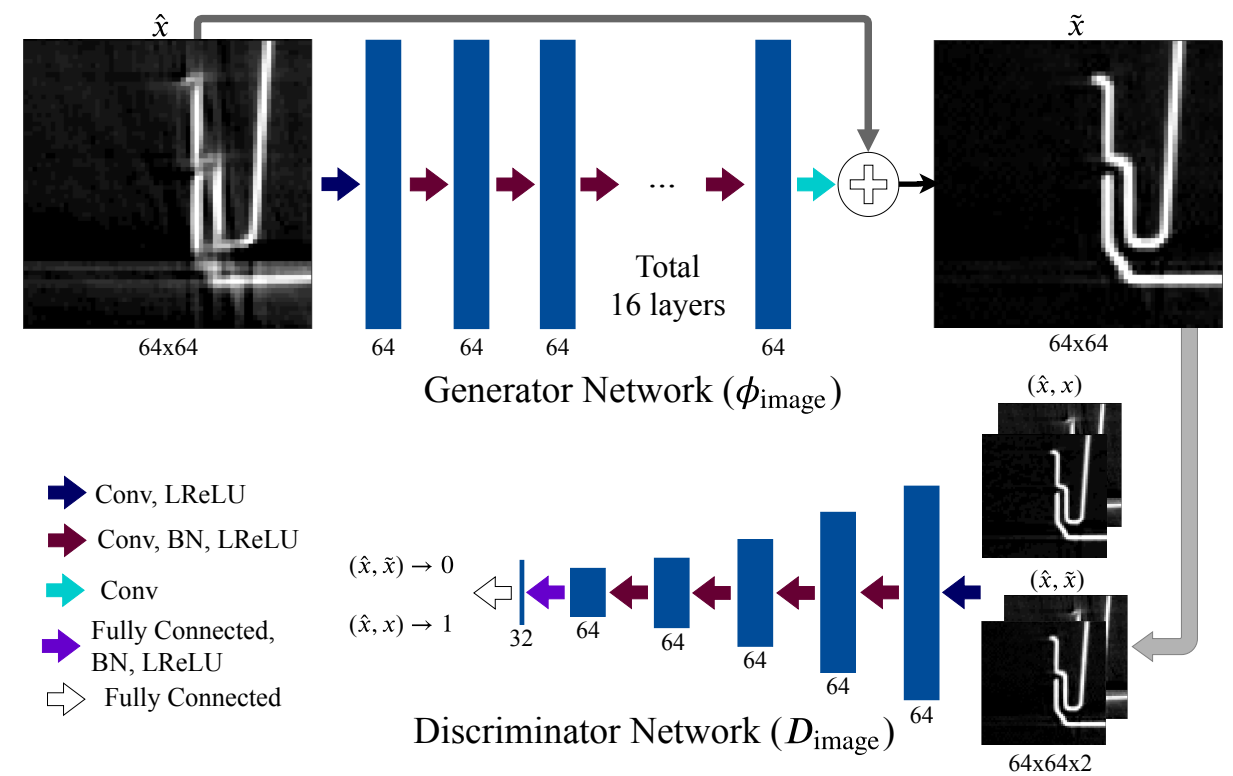

Figure 4. Overall architecture of our image-domain cGAN is presented. It learns patch-based image priors from a large security dataset. The abbreviated terms in the Figure are defined here; Conv: 2D convolution, LReLU: leaky rectified linear unit, and BN: batch-normalization.

our data-domain DNN information through the physical tomographic projection model $\mathbf{A}$.

For the second CE agent we use an image domain DNN model. In particular, we choose:

$$
F_{\text {image }}\left(v_{2}\right)=\phi_{\text {image }}\left(v_{2}\right)
$$

where $\phi_{\text {image }}$ is a DNN.

Following $[9,50]$ the solution of the CE equations can be obtained as follows. First define the vectorized aggregate variable maps:

$$
\mathbf{F}(\mathbf{v} ; \sigma)=\left(\begin{array}{c}
F_{\text {data }}\left(v_{1} ; \sigma\right) \\
F_{\text {image }}\left(v_{2}\right)
\end{array}\right)
$$

Further, define the averaging or redistribution operator G:

$$
\mathbf{G}(\mathbf{v})=\left(\begin{array}{c}
\overline{\mathbf{v}} \\
\overline{\mathbf{v}}
\end{array}\right)
$$

where $\overline{\mathbf{v}}=\sum_{i=1}^{2} \mu_{i} v_{i}$.

It can be shown that $\mathbf{z}^{*}=x^{*}-\mathbf{u}^{*}$ is a fixed point of the map $\mathbf{T}=(2 \mathbf{F}-\mathbf{I})(2 \mathbf{G}-\mathbf{I})$. Once $\mathbf{z}^{*}$ is found the CE solution can be easily computed as $x^{*}=\overline{\mathbf{z}}^{*}=\sum_{i=1}^{2} \mu_{i} z_{i}$. One way to achieve this fixed point $\mathbf{z}^{*}$ is using Mann iterations:

$$
\mathbf{z}^{(\mathbf{k}+\mathbf{1})}=(1-\rho) \mathbf{z}^{(\mathbf{k})}+\rho \mathbf{T} \mathbf{z}^{(\mathbf{k})}
$$

for all $k \geq 0$, and $\rho \in(0,1)$, where $\mathbf{z}^{(0)}$ is an initial estimate. Finally, our DICE approach that integrates data and image domain DL using CE for CT image reconstruction is provided in Algorithm 1. In this work, we simply limit

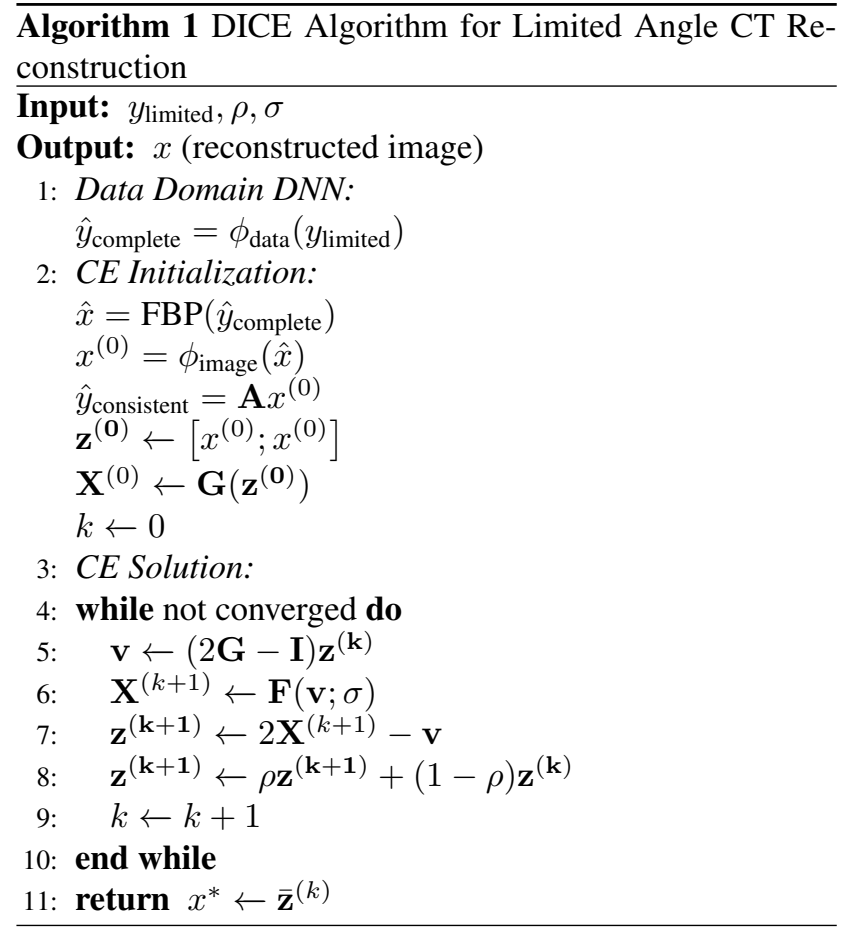

the number of outer iterations to 4 , which was observed to be adequate. Further work on convergence analysis will be conducted in the future. The application of the data agent (5) is accomplished by 20 iterations of the conjugate gradient algorithm. We use $\rho=0.25, \mu_{1}=0.6, \mu_{2}=0.4$, and $\sigma^{2}=10^{-8}$. We use Tensorflow [54] for DL components 
of our approach and ASTRA toolbox [55] for accelerated forward and back projection operations on GPU.

\section{Experiments}

We describe our experimental dataset, cGAN training strategy, and present results in this section. The real security CT dataset that we use for this work was collected using an Imatron C300 scanner as part of a data collection campaign [26]. The scans were performed with $130 \mathrm{KeV}$ peak source energy to image $475 \mathrm{~mm} \times 475 \mathrm{~mm}$ field of view. The acquired data was rebinned to a parallel beam geometry with 1024 detector channels and 720 projection angles. We split the scans into training data with 168 bags and testing data with 21 bags. For this work, we do not consider slices containing metallic objects for this work. The input and reference projection data was zero-padded to match the network input and output size.

To train the data domain cGAN we alternated between descent steps on $\phi_{\text {data }}$ and $D_{\text {data }}$. An additional $5-i$ gradient steps were performed on $D_{\text {data }}$ for $i=1,2,3,4$ epochs [53]. We used the Adam optimizer [56] with mini-batch size of 8 , and learning rate of 0.0002 , and momentum parameters $\beta_{1}=0.9, \beta_{2}=0.999$. We empirically selected the hyper-parameter to be $\lambda=100$. We trained the datadomain cGAN for 47 epochs on 31,210 examples for data completion task.

For the image domain cGAN, we used full-view MBIR [8] reconstructions as ground truth images. We apply 40 iterations of the conjugate gradient algorithm to perform $\ell_{2}$ regularized inversion using data-domain cGAN completed projection data ( $\left.\hat{y}_{\text {complete }}\right)$ and use them as low-quality input images. For the FBP + post-processing method, $90^{\circ}$ limited angle data was used to compute the low-quality input images (for training fairness). The image domain cGAN was trained on image patches corresponding to non-empty reference patches. We alternated between $\phi_{\text {image }}$ and $D_{\text {image }}$ to perform descent steps. Additional $5-i$ gradient steps were performed on $D_{\text {image }}$ for $i=1,2,3,4$ epochs [53]. We used the Adam optimizer [56] with mini-batch size of 128 , and learning rate of 0.0002 , and momentum parameters $\beta_{1}=0.9, \beta_{2}=0.999$. We empirically selected the hyper-parameter to be $\lambda=10^{-5}$. We trained the image domain cGAN for 20 epochs on 399, 823 image patches.

\subsection{Results}

First, we present data completion results along with the reference full-view projection data in Figure 5. Column (a) presents data completion results $\hat{y}_{\text {complete }}$ obtained using $\phi_{\text {data }}$ network. Column (b) presents completed projection data $\hat{y}_{\text {consistent }}$ that follows data consistency conditions. Reference full-view data is presented in column (c). Each row presents results for a different example. Due to imperfections in the data completion learning task and since data

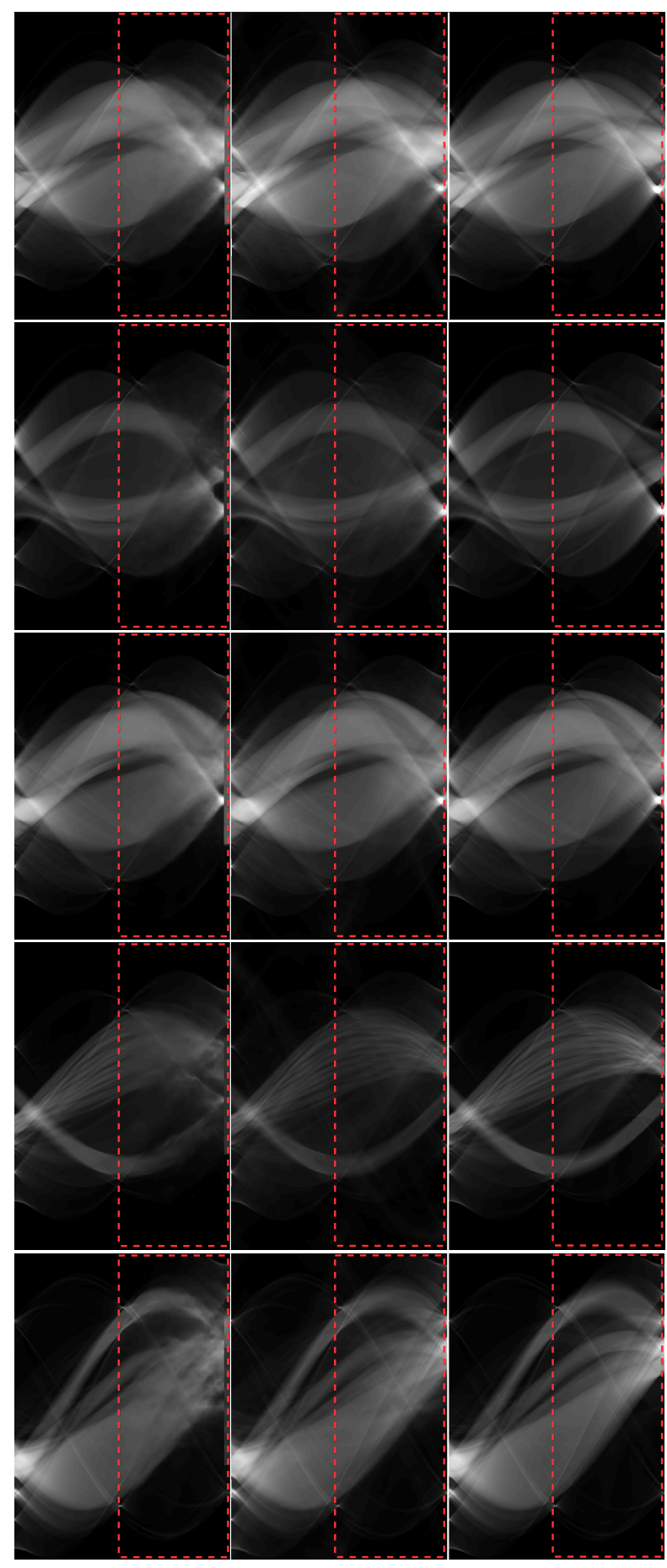
(a) $\hat{y}_{\text {complete }}$
(b) $\hat{y}_{\text {consistent }}$
(c) $y_{\text {reference }}$ Figure 5. Data completion results are presented along with reference full-view projection data. Each row presents results for a different example. Regions corresponding to completed projection data are highlighted using red rectangles.

consistency conditions are not in place, artifacts are visible in the results presented in column (a). However, since 


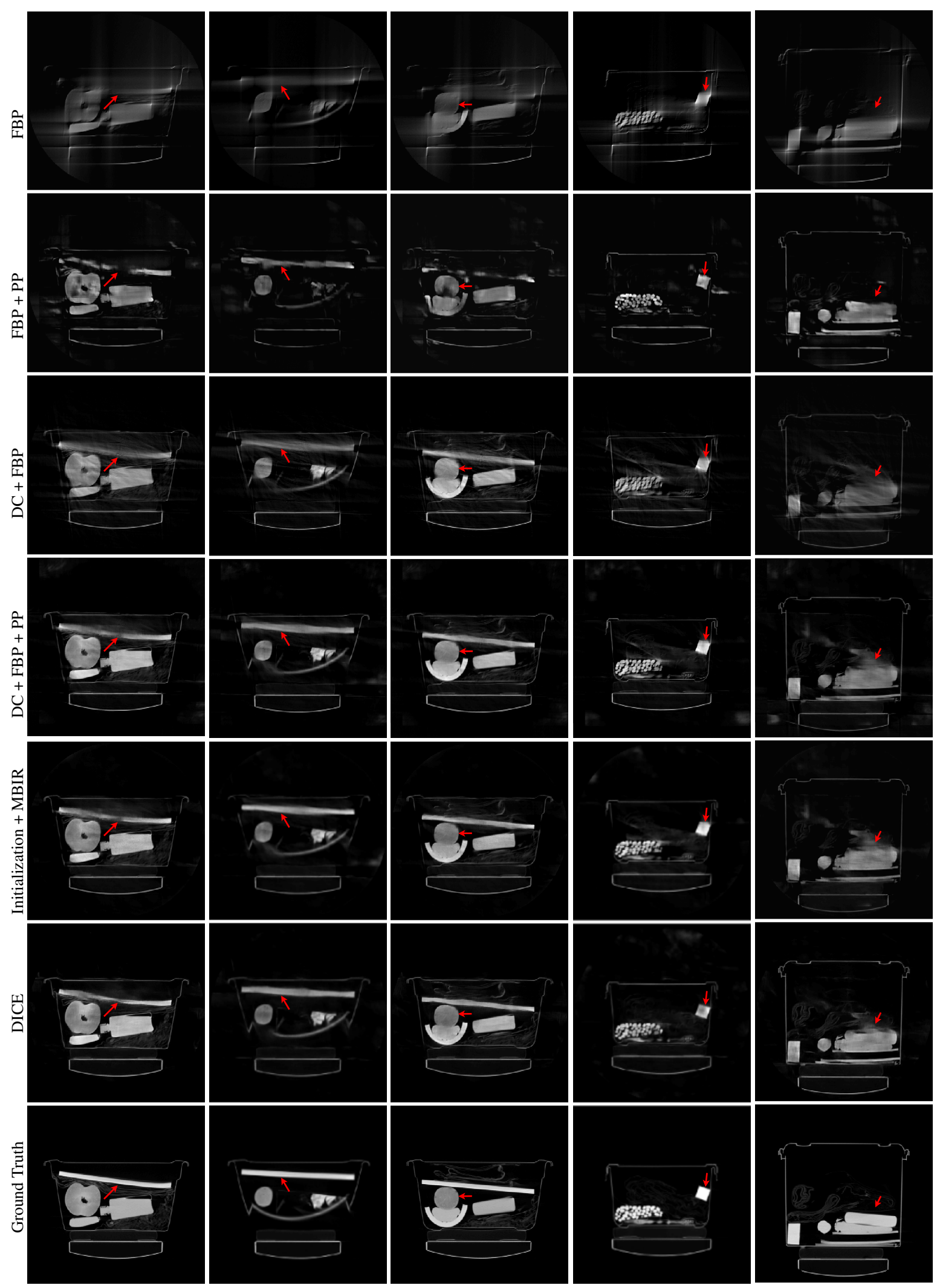

Figure 6. Limited angle CT reconstruction results using different methods are presented. Here DC refers to data domain cGAN based Data Completion, PP refers to post-processing using image domain cGAN, and Initialization refers to consistent complete data and reconstruction initialization. We use the same initialization and the same consistent completed projection data for both Initialization + MBIR and DICE methods for a fair comparison. State-of-the-art image post-processing method (second row) fails to recover lost information completely. Evidently our proposed DICE approach reconstruct superior quality images which not only recover lost information accurately but also greatly suppress image artifacts. 
Table 1. Reconstruction performance comparison of different methods on test dataset in terms of average RMSE (HU), PSNR (dB), and SSIM.

\begin{tabular}{|l|c|c|c|}
\hline Method & RMSE & PSNR & SSIM \\
\hline FBP & 137 & 20.96 & 0.50 \\
\hline FBP+PP & 115 & 22.46 & 0.44 \\
\hline DC + FBP & 95 & 24.18 & 0.73 \\
\hline DC + FBP + PP & 79 & 25.86 & 0.72 \\
\hline Initialization + MBIR & 76 & 26.14 & 0.78 \\
\hline DICE (proposed) & $\mathbf{7 3}$ & $\mathbf{2 6 . 5 5}$ & $\mathbf{0 . 8 1}$ \\
\hline
\end{tabular}

$\hat{y}_{\text {consistent }}$ follows data consistency conditions, the results in column (b) are visually more plausible.

Limited angle CT images reconstructed using different methods are presented in Figure 6. Each column presents results for a different example, corresponding to the same examples considered in rows of Figure 5. The FBP reconstructions in the first row have large areas of lost structure as well as streaking artifacts. DNN post-processing of the FBP images in the second row serves to recover some of the lost structure, but fails to correct CT numbers and much structure is still mot recovered. Data domain DL aims to recover structure by learning to complete projection data prior to image reconstruction. The third row shows conventional reconstruction of this completed data. While it does a good job recovering lost structure, artifacts can still be seen. The fourth row shows post-processing of these completed data conventional reconstructions, which can be seen to result in slight improvements, such as intensity uniformity. The fifth row shows the results of combining consistent completed data and reconstruction initialization with 4 iterations of MBIR [8], which produces minor improvements. Our proposed integrated data and image domain DNN method is shown in the sixth row. The reconstruction produces more compact object shapes consistent with the ground truth and more uniform CT numbers. The reference images are shown in the bottom row.

Finally, we also perform quantitative analysis on 315 slices where ground truth images are full-view MBIR reconstructions [8] in Table 1. We consider three metrics RMSE: root mean square error (HU), PSNR: peak signal-to-noise ratio, and SSIM: structural similarity index. Our DICE approach involving data and image domain DL using CE framework outperforms all the considered methods on all three metrics. In our analysis, DICE reconstruction results are not heavily dependent upon initialization. Data completion alone combined with FBP significantly outperforms image post-processing approach, suggesting the value of the part of the process to the overall result.

\section{Conclusion}

In this work we presented DICE framework integrating both data-domain and image-domain deep learning using consensus equilibrium. Our motivation was to solve challenging limited angle CT problems. We demonstrated the approach on a limited angle security CT data set comparing the method to a variety of alternatives and showing superior results. This initial work demonstrates the potential value of combining deep learning in these two complementary domains. Ongoing work is aimed at folding our data-domain models directly into the CE framework.

\section{Acknowledgement}

This material is based upon work supported by the U.S. Department of Homeland Security, Science and Technology Directorate, Office of University Programs, under Grant Award 2013-ST-061ED0001. The views and conclusions contained in this document are those of the authors and should not be interpreted as necessarily representing the official policies, either expressed or implied, of the U.S. Department of Homeland Security.

\section{Appendix 1: cGAN Objective Function}

The overall cGAN cost function consists of two terms: a conventional pixel based loss term and an adversarial loss term. Isola et al. [14] reported that using such a combination results in better performance. Generator and discriminator networks in our cGANs are jointly trained by optimizing a mini-max cost function [14, 52]:

$$
\begin{aligned}
\phi_{i}^{*} & =\arg \min _{\phi_{i}} \max _{D_{i}} \mathcal{L}_{c G A N}\left(\phi_{i}, D_{i}\right) \\
& +\lambda \mathbb{E}_{I n, G T}\left[\left\|G T-\phi_{i}(I n)\right\|_{2}^{2}\right]
\end{aligned}
$$

where In and GT represent input and ground truth used for network training, $\phi_{i}($ In $)$ represents output of $i^{t h}$ generator network, $D_{i}$ represents $i^{\text {th }}$ discriminator network, $\lambda$ is a hyper-parameter used to control the contribution of both terms in the overall cost function, and $\mathbb{E}_{I n, G T}$ describes expectation over input and output pair dataset density. Both networks $\phi_{i}$ and $D_{i}$ play a mini-max game by acting adversaries $-\phi_{i}$ efforts to output images similar to $G T$, and $D_{i}$ learns to differentiate between $\left(\operatorname{In}, \phi_{i}(\operatorname{In})\right)$ and $(I n, G T)$ pairs. This interaction is captured by the first term $\mathcal{L}_{c G A N}\left(\phi_{i}, D_{i}\right)$ of overall cost function, and is defined as:

$$
\begin{aligned}
\mathcal{L}_{c G A N}\left(\phi_{i}, D_{i}\right) & =\mathbb{E}_{I n, G T}[\log D(\operatorname{In}, G T)] \\
& +\mathbb{E}_{\text {In }}\left[\log \left(1-D\left(\operatorname{In}, \phi_{i}(\operatorname{In})\right)\right]\right.
\end{aligned}
$$

where $\mathbb{E}_{\text {In }}$ represents input dataset density. We use the $\ell_{2}$ weight regularization on the generator network for efficient training. Additionally, we use one-sided label smoothing as suggested by Salimans et al. [57].

\section{References}

[1] Elbakri, Idris A and Fessler, Jeffrey A, "Statistical image reconstruction for polyenergetic X-ray computed tomography," IEEE transactions on medical imaging, vol. 21, no. 2, pp. 89-99, 2002. 
[2] Chang, Zhiqian and Zhang, Ruoqiao and Thibault, JeanBaptiste and Pal, Debashish and Fu, Lin and Sauer, Ken and Bouman, Charles, "Modeling and pre-treatment of photonstarved CT data for iterative reconstruction," IEEE transactions on medical imaging, vol. 36, no. 1, pp. 277-287, 2017.

[3] Tuysuzoglu, Ahmet and Khoo, Yuehaw and Karl, W Clem, "Variable splitting techniques for discrete tomography," in Image Processing (ICIP), 2016 IEEE International Conference on. IEEE, 2016, pp. 1764-1768.

[4] K. A. Mohan, S. Venkatakrishnan, J. W. Gibbs, E. B. Gulsoy, X. Xiao, M. De Graef, P. W. Voorhees, and C. A. Bouman, "TIMBIR: A Method for Time-Space Reconstruction from Interlaced Views," IEEE Transactions on Computational Imaging, vol. 1, no. 2, pp. 96-111, 2015.

[5] S. Carmignato, W. Dewulf, and R. Leach, Industrial X-ray computed tomography. Springer, 2018.

[6] Martin, Limor and Tuysuzoglu, Ahmet and Karl, W Clem and Ishwar, Prakash, "Learning-Based Object Identification and Segmentation Using Dual-Energy CT Images for Security," IEEE Transactions on Image Processing, vol. 11, no. 24, pp. 4069-4081, 2015.

[7] M. U. Ghani and W. C. Karl, "Deep learning based sinogram correction for metal artifact reduction," Electronic Imaging, no. XVI, pp. 472-1 - 472-8, 2018.

[8] Jin, Pengchong and Bouman, Charles A and Sauer, Ken D, "A Model-Based Image Reconstruction Algorithm With Simultaneous Beam Hardening Correction for X-Ray CT." IEEE Trans. Computational Imaging, vol. 1, no. 3, pp. 200$216,2015$.

[9] G. T. Buzzard, S. H. Chan, S. Sreehari, and C. A. Bouman, "Plug-and-play unplugged: Optimization-free reconstruction using consensus equilibrium," SIAM Journal on Imaging Sciences, vol. 11, no. 3, pp. 2001-2020, 2018.

[10] L. Ritschl, F. Bergner, C. Fleischmann, and M. Kachelrieß, "Improved total variation-based CT image reconstruction applied to clinical data," Physics in Medicine \& Biology, vol. 56, no. 6, p. 1545, 2011.

[11] R. Zhang, D. H. Ye, D. Pal, J.-B. Thibault, K. D. Sauer, and C. A. Bouman, "A Gaussian mixture MRF for model-based iterative reconstruction with applications to low-dose X-ray CT," IEEE Transactions on Computational Imaging, vol. 2, no. 3, pp. 359-374, 2016.

[12] Ye, Dong Hye and Srivastava, Somesh and Thibault, JeanBaptiste and Sauer, Ken and Bouman, Charles, "Deep Residual Learning for Model-Based Iterative CT Reconstruction using Plug-and-Play Framework," in 2018 IEEE International Conference on Acoustics, Speech and Signal Processing (ICASSP). IEEE, 2018, pp. 6668-6672.

[13] C. J. PeUizzari, M. F. Spencer, and C. A. Bouman, "Coherent-Image Reconstruction Using Convolutional Neural Networks," in Mathematics in Imaging. Optical Society of America, 2019, pp. MTu4D-4.
[14] P. Isola and J. Y. Zhu and T. Zhou and A. A. Efros, "Image-to-Image Translation with Conditional Adversarial Networks," in 2017 IEEE Conference on Computer Vision and Pattern Recognition (CVPR), 2017-07, pp. 5967-5976.

[15] J. H. Cho and J. A. Fessler, "Motion-compensated image reconstruction for cardiac CT with sinogram-based motion estimation," in 2013 IEEE Nuclear Science Symposium and Medical Imaging Conference (2013 NSS/MIC). IEEE, 2013, pp. 1-5.

[16] A. Tuysuzoglu, Y. Khoo, and W. C. Karl, "Fast and robust discrete computational imaging," Electronic Imaging, vol. 2017, no. 17, pp. 49-54, 2017.

[17] Y. Arai, K. Honda, K. Iwai, and K. Shinoda, "Practical model 3DX of limited cone-beam X-ray CT for dental use," in International Congress Series, vol. 1230. Elsevier, 2001, pp. 713-718.

[18] L. T. Niklason, B. T. Christian, L. E. Niklason, D. B. Kopans, D. E. Castleberry, B. Opsahl-Ong, C. E. Landberg, P. J. Slanetz, A. A. Giardino, R. Moore et al., "Digital tomosynthesis in breast imaging," Radiology, vol. 205, no. 2, pp. 399-406, 1997.

[19] Krizhevsky, Alex and Sutskever, Ilya and Hinton, Geoffrey E, "Imagenet classification with deep convolutional neural networks," in Advances in neural information processing systems, 2012, pp. 1097-1105.

[20] Xie, Junyuan and Xu, Linli and Chen, Enhong, "Image denoising and inpainting with deep neural networks," in $A d$ vances in Neural Information Processing Systems, 2012, pp. 341-349.

[21] Köhler, Rolf and Schuler, Christian and Schölkopf, Bernhard and Harmeling, Stefan, "Mask-specific inpainting with deep neural networks," in German Conference on Pattern Recognition. Springer, 2014, pp. 523-534.

[22] Kim, Jiwon and Kwon Lee, Jung and Mu Lee, Kyoung, "Accurate image super-resolution using very deep convolutional networks," in Proceedings of the IEEE Conference on Computer Vision and Pattern Recognition, 2016, pp. 1646-1654.

[23] Zhang, Kai and Zuo, Wangmeng and Chen, Yunjin and Meng, Deyu and Zhang, Lei, "Beyond a gaussian denoiser: Residual learning of deep cnn for image denoising," IEEE Transactions on Image Processing, 2017.

[24] Zhao, Hang and Gallo, Orazio and Frosio, Iuri and Kautz, Jan, "Loss functions for image restoration with neural networks," IEEE Transactions on Computational Imaging, vol. 3, no. 1, pp. 47-57, 2017.

[25] Chaudhury, Subhajit and Roy, Hiya, "Can fully convolutional networks perform well for general image restoration problems?" in Machine Vision Applications (MVA), 2017 Fifteenth IAPR International Conference on. IEEE, 2017, pp. 254-257. 
[26] Crawford, C, "Advances in automatic target recognition (ATR) for CT based object detection system-Final report, Dept," Homeland Security Center Excellence, Task order number HSHQDC-12-J-00429, Boston, MA, 2014.

[27] S. Ravishankar, J. C. Ye, and J. A. Fessler, "Image reconstruction: From sparsity to data-adaptive methods and machine learning," arXiv preprint arXiv:1904.02816, 2019.

[28] H. Zhang, L. Li, K. Qiao, L. Wang, B. Yan, L. Li, and G. Hu, "Image prediction for limited-angle tomography via deep learning with convolutional neural network," arXiv preprint arXiv:1607.08707, 2016.

[29] J. Gu and J. C. Ye, "Multi-scale wavelet domain residual learning for limited-angle CT reconstruction," arXiv preprint arXiv:1703.01382, 2017.

[30] Z. Li, W. Zhang, L. Wang, A. Cai, N. Liang, B. Yan, and $\mathrm{L} . \mathrm{Li}$, "A sinogram inpainting method based on generative adversarial network for limited-angle computed tomography," in 15th International Meeting on Fully ThreeDimensional Image Reconstruction in Radiology and $\mathrm{Nu}$ clear Medicine, vol. 11072. International Society for Optics and Photonics, 2019, p. 1107220.

[31] Anirudh, Rushil and Kim, Hyojin and Thiagarajan, Jayaraman $\mathbf{J}$ and Mohan, K Aditya and Champley, Kyle and Bremer, Timo, "Lose The Views: Limited Angle CT Reconstruction via Implicit Sinogram Completion," arXiv preprint arXiv:1711.10388, 2017.

[32] T. Würfl, M. Hoffmann, V. Christlein, K. Breininger, Y. Huang, M. Unberath, and A. K. Maier, "Deep learning computed tomography: Learning projection-domain weights from image domain in limited angle problems," IEEE transactions on medical imaging, vol. 37, no. 6, pp. 1454-1463, 2018.

[33] Jin, Kyong Hwan and McCann, Michael T and Froustey, Emmanuel and Unser, Michael, "Deep convolutional neural network for inverse problems in imaging," IEEE Transactions on Image Processing, vol. 26, no. 9, pp. 4509-4522, 2017.

[34] Yang, Qingsong and Yan, Pingkun and Zhang, Yanbo and Yu, Hengyong and Shi, Yongyi and Mou, Xuanqin and Kalra, Mannudeep K and Wang, Ge, "Low dose CT image denoising using a generative adversarial network with wasserstein distance and perceptual loss," arXiv preprint arXiv:1708.00961, 2017.

[35] Li, Heyi and Mueller, Klaus, "Low-dose ct streak artifacts removal using deep residual neural network," in Proceedings of Fully 3D conference, 2017, pp. 191-194.

[36] Han, Yoseob and Ye, Jong Chul, "Framing U-Net via deep convolutional framelets: Application to sparse-view CT," IEEE transactions on medical imaging, vol. 37, no. 6, pp. 1418-1429, 2018.
[37] H. Lee, J. Lee, H. Kim, B. Cho, and S. Cho, "Deep-NeuralNetwork-Based Sinogram Synthesis for Sparse-View CT Image Reconstruction," IEEE Transactions on Radiation and Plasma Medical Sciences, vol. 3, no. 2, pp. 109-119, 2018.

[38] Ghani, Muhammad Usman and Karl, W Clem, "Deep Learning-Based Sinogram Completion for Low-Dose CT," in 2018 IEEE 13th Image, Video, and Multidimensional Signal Processing Workshop (IVMSP). IEEE, 2018, pp. 1-5.

[39] X. Dong, S. Vekhande, and G. Cao, "Sinogram interpolation for sparse-view micro-ct with deep learning neural network," in Medical Imaging 2019: Physics of Medical Imaging, vol. 10948. International Society for Optics and Photonics, 2019, p. 1094820.

[40] K. Liang, H. Yang, and Y. Xing, "Comparision of projection domain, image domain, and comprehensive deep learning for sparse-view X-ray CT image reconstruction," arXiv preprint arXiv:1804.04289, 2018.

[41] B. Zhu, J. Z. Liu, S. F. Cauley, B. R. Rosen, and M. S. Rosen, "Image reconstruction by domain-transform manifold learning," Nature, vol. 555, no. 7697, p. 487, 2018.

[42] J. He and J. Ma, "Radon inversion via deep learning," in Medical Imaging 2019: Physics of Medical Imaging, vol. 10948. International Society for Optics and Photonics, 2019, p. 1094810.

[43] S. V. Venkatakrishnan, C. A. Bouman, and B. Wohlberg, "Plug-and-play priors for model based reconstruction," in 2013 IEEE Global Conference on Signal and Information Processing. IEEE, 2013, pp. 945-948.

[44] S. Sreehari, S. V. Venkatakrishnan, B. Wohlberg, G. T. Buzzard, L. F. Drummy, J. P. Simmons, and C. A. Bouman, "Plug-and-play priors for bright field electron tomography and sparse interpolation," IEEE Transactions on Computational Imaging, vol. 2, no. 4, pp. 408-423, 2016.

[45] T. Meinhardt, M. Moller, C. Hazirbas, and D. Cremers, "Learning proximal operators: Using denoising networks for regularizing inverse imaging problems," in Proceedings of the IEEE International Conference on Computer Vision, 2017, pp. 1781-1790.

[46] S. Ono, "Primal-dual plug-and-play image restoration," IEEE Signal Processing Letters, vol. 24, no. 8, pp. 11081112, 2017.

[47] U. S. Kamilov, H. Mansour, and B. Wohlberg, "A plug-andplay priors approach for solving nonlinear imaging inverse problems," IEEE Signal Processing Letters, vol. 24, no. 12, pp. 1872-1876, 2017.

[48] H. Gupta, K. H. Jin, H. Q. Nguyen, M. T. McCann, and M. Unser, "Cnn-based projected gradient descent for consistent ct image reconstruction," IEEE transactions on medical imaging, vol. 37, no. 6, pp. 1440-1453, 2018. 
[49] Y. Romano, M. Elad, and P. Milanfar, "The little engine that could: Regularization by denoising (RED)," SIAM Journal on Imaging Sciences, vol. 10, no. 4, pp. 1804-1844, 2017.

[50] C. A. Bouman, G. T. Buzzard, and S. H. Chan, "Consensus equilibrium: A framework for model integration," in Imaging and Applied Optics 2018 (3D, AO, AIO, COSI, DH, IS, LACSEA, LS\&C, MATH, pcAOP). Optical Society of America, 2018, p. MW5D.1. [Online]. Available: http://www.osapublishing.org/abstract.cfm? URI=MATH-2018-MW5D.1

[51] J. L. Prince and A. S. Willsky, "Constrained sinogram restoration for limited-angle tomography," Optical Engineering, vol. 29, no. 5, pp. 535-545, 1990.

[52] Goodfellow, Ian and Pouget-Abadie, Jean and Mirza, Mehdi and $\mathrm{Xu}$, Bing and Warde-Farley, David and Ozair, Sherjil and Courville, Aaron and Bengio, Yoshua, "Generative adversarial nets," in Advances in neural information processing systems, 2014, pp. 2672-2680.

[53] Ghani, Muhammad Usman and Karl, W Clem, "Fast Enhanced CT Metal Artifact Reduction using Data Domain Deep Learning," IEEE Transactions on Computational Imaging, 2019.

[54] Abadi, Martín and Agarwal, Ashish and Barham, Paul and Brevdo, Eugene and Chen, Zhifeng and Citro, Craig and Corrado, Greg S and Davis, Andy and Dean, Jeffrey and Devin, Matthieu and others, "Tensorflow: Large-scale machine learning on heterogeneous distributed systems," arXiv preprint arXiv:1603.04467, 2016.

[55] van Aarle, Wim and Palenstijn, Willem Jan and Cant, Jeroen and Janssens, Eline and Bleichrodt, Folkert and Dabravolski, Andrei and De Beenhouwer, Jan and Batenburg, K Joost and Sijbers, Jan, "Fast and flexible X-ray tomography using the ASTRA toolbox," Optics express, vol. 24, no. 22, pp. 25 129-25 147, 2016.

[56] Kingma, Diederik and Ba, Jimmy, "Adam: A method for stochastic optimization," arXiv preprint arXiv:1412.6980, 2014.

[57] T. Salimans, I. Goodfellow, W. Zaremba, V. Cheung, A. Radford, and X. Chen, "Improved techniques for training gans," in Advances in neural information processing systems, 2016, pp. 2234-2242. 